\title{
Peripheral axotomy-induced changes of motor function and histological structure of spinal anterior horn
}

Amir Raoofi (1), Mohammad Amin Abdollahifar (1), Abbas Aliaghaei (1), Abbas Piryaei (1), Fatemeh Hejazi (2), Ensieh Sajadi (1), Ali Rashidiani-Rashidabadi (1), Yousef Sadeghi (1)

(1) Department of Biology and Anatomical Sciences, School of Medicine, Shahid Beheshti University of Medical Sciences, Tehran, Iran; (2) Department of Polymer Engineering and Color Technology, Amirkabir University of Technology (Tehran Polytechnic), Tehran, Iran

This article is distributed under the terms of the Creative Commons Attribution Noncommercial License (CC BY-NC 4.0) which permits any noncommercial use, distribution, and reproduction in any medium, provided the original author(s) and source are credited.

\begin{abstract}
The aim of this study was to evaluate changes of both peripheral motor function and histology of spinal anterior horn in adult rats after unilateral sciatectomy. Ten adult healthy rats served as control group, while in the ten rat experimental group the right sciatic nerve was severed. We followed-up nerve motor function using a sciatic function index and electromyography activity of the gastrocnemious muscle. The rats of the experimental group presented the expected gross locomotor deficit and leg muscle atrophy. At 12 weeks post sciatectomy, L4 and L5 spinal cord segments were removed from the twenty rats and were analysed by istological stereological methods. In the axotomized animals volume of the anterior horn and its content of motor neurons decreased, while the content of astrocytes increased $(\mathrm{p}<0.05)$. Thus, in adult rats, beside the obvious peripheral nerve disfuction, the sciatic nerve axotomy have severe consequences on the soma of the injured motor neurons in the spinal anterior horn. All these quantitative analyses may be usefull to quantify changes occurring in adult animals after axotomy and eventual management to modify the final outcomes in peripheral nerve disorders.
\end{abstract}

Key Words: Peripheral axotomy, motor function, anterior horn, stereology, degeneration

Eur J Transl Myol 29 (1): 33-40, 2019

Severe nerve injury may have devastating impact on quality of life. Axotomy, a peripheral nerve injury (PNI), may occur by trauma and many more medical disorders. ${ }^{1}$ Typical symptoms are sensory and motor function deficts that may result in complete paralysis of affected limb or in intractable neuropathic pain. ${ }^{2}$ Most of the peripheral nerves, particularly spinal nerves, are mixed nerves that contain motor and sensory nerve fibers. ${ }^{3,4}$ Though damaged nerve may regenerate following peripheral axotomy, some of the dorsal root ganglion and ventral horn neurons undergo a series of retrograde degenerative changes that may lead to neuronal death. ${ }^{5-8}$ These changes are related to dysregulation of the retrograde flow of neurotrophic factors from periphery to the neurons. ${ }^{9}$ Albeit different type of graft including autografts, allografts, and xenografts have been used to increase nerve regeneration, they present limited potentials of recovery and immunological rejection. ${ }^{10,11}$ Previous studies have shown that, following injury, motoneuron degeneration in the spinal cord is mediated by oxidative stress and apoptosis, possibly related to trophic factor deprivation, as well as by activation of death receptors. ${ }^{12}$ As for PNI, motor functional recovery, the end-goal for patients, depends on successful axonal regeneration of the motoneurons, ${ }^{3,4}$ that is too often quite slow and partial. ${ }^{13}$ Therefore, we are interested to set up a standard animal model to study impairment of motor function and of histological consequences on the spinal anterior horn induced by peripheral axotomy. Here, we present the main changes occurring at functional and structural levels in the classical rat model of unilateral sciatic nerve resection.

\section{Materials and Methods}

\section{Animals and surgical procedures}

Twenty adult male Wistar rats that weighed approximately $260 \mathrm{~g}$ were used. Animals were housed individually in standard rat cages $20 \times 20 \times 40 \mathrm{~cm}$ in size in the animal house (12-hs light / 12-hs dark environment), and provided with water ad libitum. Rats were randomized into two groups of ten animals: 1. Normal 
group: healthy rats; 2 . Axotomy group: rats with severed sciatic nerve without further treatments. Briefly, animals were anesthetized by intraperitoneal injection of sodium pentobarbital (40 mg/kg body weight) before the sciatic nerve was exposed by a $2 \mathrm{~cm}$ longitudinal skin incision then interrupted at $0.5 \mathrm{~cm}$ below ischial tuberosity in the right lateral thigh. A nerve segment (10 $\mathrm{mm}$ in lenght) was removed. After surgery, animals were housed in their cages and fed routinely under normal conditions.

\section{Sciatic Function Index}

Four, eight and twelve weeks post-surgery, the walking pattern of animals were recorded for the analysis of the sciatic functional index (SFI). Briefly, before the experiments, rats were trained to walk down a wooden track $(100 \times 20 \times 15 \mathrm{~cm})$ into a darkened goal box. After surgery, the animal's hind paws were dipped using inkpad and the changes in their paw prints that resulted from nerve denervation were recorded. The recordings continued until five measurable footprints were collected. From the footprints, the following parameters were calculated using a ruler: print length (PL), that refers to the distance from the heel to the top of the third toe, intermediary toe spread (ITS) that refers to distance from the second to the fourth toe, and toe spread the distance between the first and the fifth toe. All these measurements were obtained both from the right experimental foot (EPL, ETS and EITS, respectively) and from the left non- operated foot (NPL, NTS and NITS, respectively) of each rat. By using these data, SFI was calculated by the equation of Table $1 .{ }^{14}$

\section{Electromyographic studies (EMG)}

After 12 week of sciatic nerve axotomy, EMG evaluation was performed on all rats prior to sacrifice. Under anesthesia by intraperitoneally xylazine $(8 \mathrm{mg} / \mathrm{k})$ and ketamine hydrochloride $(60 \mathrm{mg} / \mathrm{kg})$, the right sciatic nerve of the operated side was re-exposed by longitudinal incision on the posterior aspect of each thigh from the greater trochanter to the knee. A bipolar stimulating electrode was positioned to the nerve trunk at a location $10 \mathrm{~mm}$ proximal to the site of axotomy and compound muscle action potentials were recorded in the belly of the gastrocnemius muscle at the ipsilateral side using an EMG recorder. The physiologic parameters including the peak amplitude of compound action potentials and latency were measured. ${ }^{15}$

\section{Perfusion and tissue collection}

After 12 weeks, animals were anesthetized with ketamine $(100 \mathrm{mg} / \mathrm{kg})$ and xylazine $(10 \mathrm{mg} / \mathrm{kg})$ intraperitoneally, and then rats were perfused intracardially with $150 \mathrm{ml}$ normal saline and $200 \mathrm{ml}$ 4\% paraformaldehyde. Spinal cord samples were extracted and post-fixed in $4 \%$ paraformaldehyde at $48 \mathrm{C}$ for $4-5$ days. The paraffin blocks were prepared and cut in serial sections of $10 \mu \mathrm{m}$ thickness. Sections were stained with cresyl violet.

\section{Cresyl violet staining of spinal cord}

Transverse sections were re-hydrated in $70 \%, 95 \%, 95 \%$, $100 \%$, and $100 \%$ ethanol (3 min each). After a 1-min wash in water, the slides were incubated in $0.25 \%$ cresyl violet in $200 \mathrm{mM}$ acetate buffer for $30 \mathrm{~min}$. Following 10 dips each in water, $70 \%$ and $100 \%$ ethanol, the tissue was differentiated in $0.25 \%$ glacial acetic acid until white matter was visible. Then slides were dipped in $95 \%$ and $100 \%$ ethanol (10 dips each), and cover-slipped using Permount (Fisher Scientific, Unionville, Canada), were slides viewed by light microscope (Nikon, Japan).

Motoneurons were identified by their position in the spinal cord (ventral horn, lamina IX), size (substantially larger than interneurons and glial cells), and presence of Nissl substance arranged in polygonal clumps. The motoneurons present in the ventral horn on the ipsilateral and contralateral sides to the injury were counted in alternate sections of each specimen in the injured area of the lumbar intumescence. Only cells with visible nuclei were counted.

\section{Immunohistochemical study of GFAP expression in the anterior horn of spinal cord}

Rats were deeply anesthetized by chloral hydrate and perfused transcardially using chilled saline followed by a fixative consisting of $4 \%$ paraformaldehyde in $0.1 \mathrm{M}$ PBS. Then, spinal cord were placed in formalin, prepared, and placed on slides. The primary antibody was diluted with PBS containing $0.3 \%$ Triton X-100 and $1 \%$ bovine serum albumin (BSA). Sections were incubated in primary antibodies against GFAP (1:300) overnight in 4 ${ }^{\circ} \mathrm{C}$. Sections were then incubated with the avidin- biotin complex substrate and treated with $0.05 \%$ 3,3diaminobenzidine tetrahydrochloride and $0.03 \%$ hydrogen peroxide in 0.05 mTris buffer ( $\mathrm{pH} 7.6)$. After immunohistochemical reaction, sections were mounted, counterstained, and observed under a light microscope.

Table 1. Sciatic function index measuremet. Using the measured values, SFI was calculated by the following equation. ${ }^{14}$

$$
\mathrm{SFI}=-38.3(\mathrm{EPL}-\mathrm{NPL}) / \mathrm{NPL}+109.5(\mathrm{ETS}-\mathrm{NTS}) / \mathrm{NTS}+13.3(\mathrm{EIT}-\mathrm{NIT}) / \mathrm{NIT}-8.8
$$

SFI value of nearly 0 represents a normal functional leg.

The SFI value of -100 indicates total dysfunction of the sciatic nerve. 

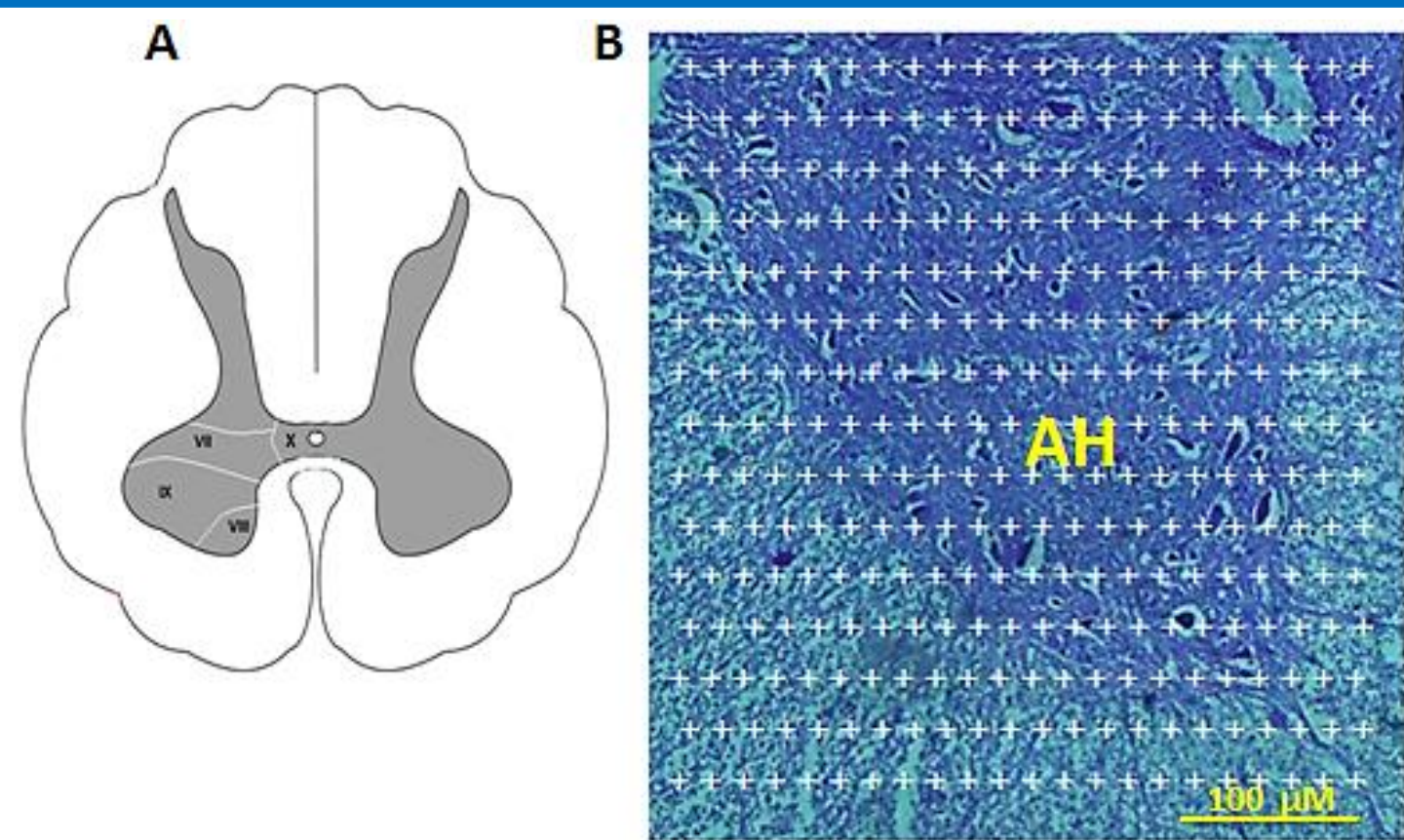

Fig 1. Volume study. (A, B) Schematic drawings of transverse section from L5 level of the spinal cord in control and axotomy groups. Photomicrograph of the spinal cord stained with Cresyl violet. $\times 10$. A grid of points was superimposed on the Image for estimation of total volume of anterior horn. AH, Anterior horn.

Stereological estimate of the total volume of spinal anterior horn

The volume was estimated using the Cavalieri method. Briefly, 10 sections were selected using systematic, uniformly random sampling for stereological estimations. Each microscopic slide was analyzed using a video microscopy system which was made up of a microscope (E-200, Nikon, Tokyo, Japan) linked to a video camera, a computer, a flat monitor, and a microcator (MT-12, Heidenhain, Traunreut, Germany). The formula for calculation of volume of spinal anterior horn was as follows, (Figure 1): ${ }^{16}$
$\mathrm{V}_{\text {spinal anterior horn }}=\sum \mathrm{P} \times \mathrm{d} \times \frac{\mathrm{a}}{\mathrm{p}}$

Where " $\Sigma \mathrm{P}$ " was the total points hitting the anterior horn sections, "a/p" was the area associated with each point, and " $d$ " was the distance between the sampled sections.

Stereological estimate of the number of motor neurons and astrocytes cells

The optical dissector method was used to determine the total number of neurons and astrocytes cells. Random sampling was ensured by moving the microscopic field position at equal intervals. A microcator was used for
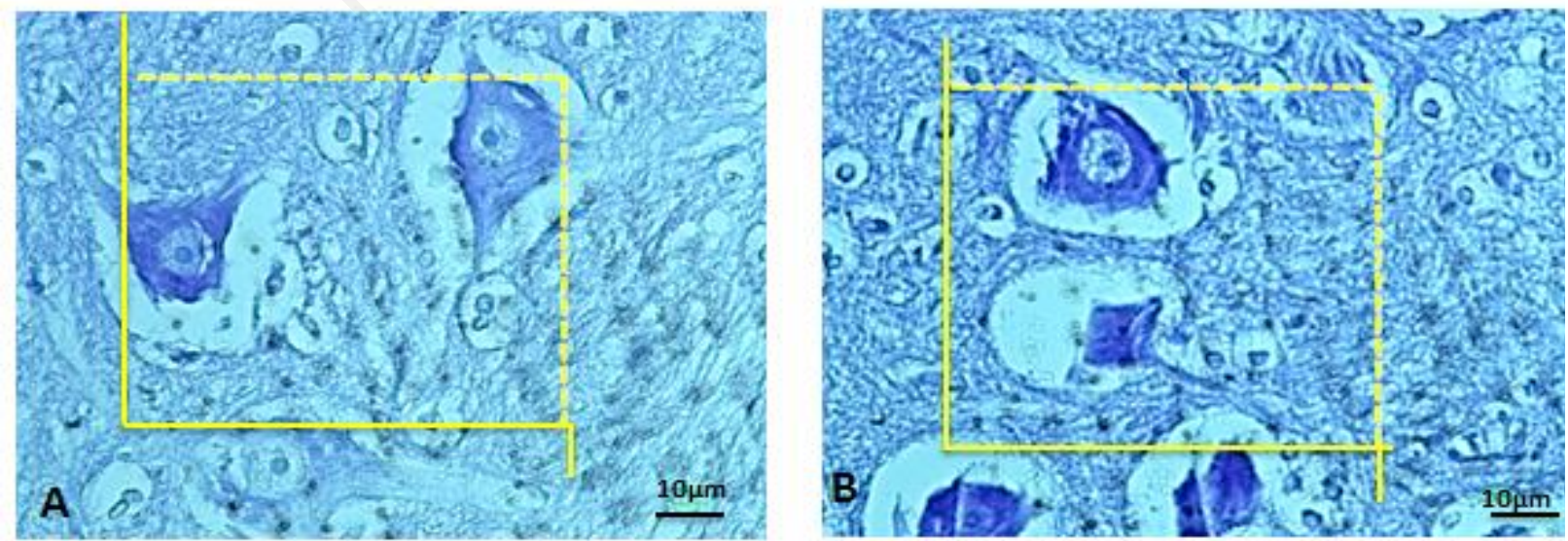

Fig 2. Photomicrographs of the spinal cord stained with cresyl violet. $\times 40$. Counting frames were superimposed on Images for estimation of total number of anterior horn neurons. A: control and B: axotomy. 
A

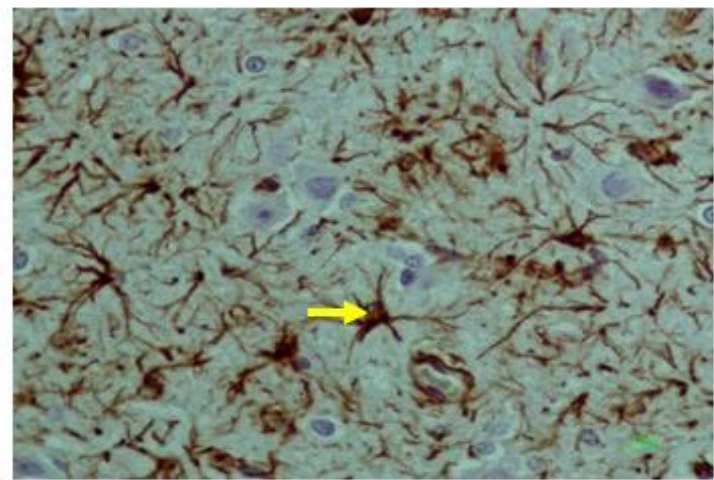

B

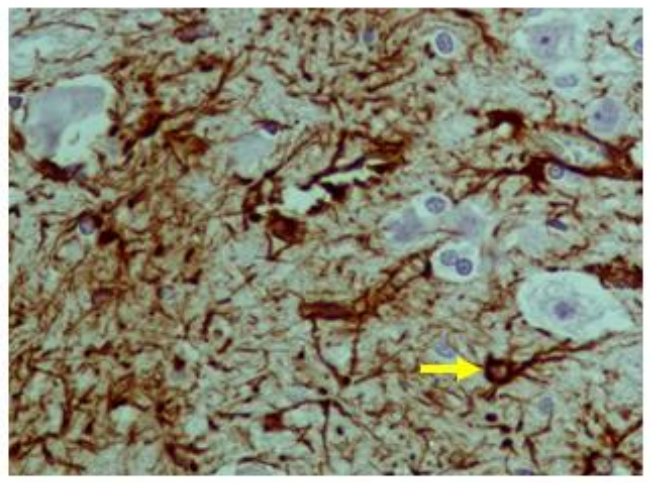

Fig 3. Astrocytic migration (gliosis). Immunohistochemistry against GFAP was done among various groups.in the anterior horn of spinal cord. $\times 40$. A: control and B: axotomy. Astrocyte (arrow).

measurement of the $\mathrm{Z}$-axis movement of the microscope stage. An unbiased counting frame, with exclusion and inclusion borders, was superimposed, according to the sectional images, which were observed on the monitor. A nucleus was counted if it fell completely or partially within the counting frame, and did not reach the exclusion line. The formula for calculation of numerical density (Nv) was as follows ${ }^{16}$ (Figure 2 and 3):

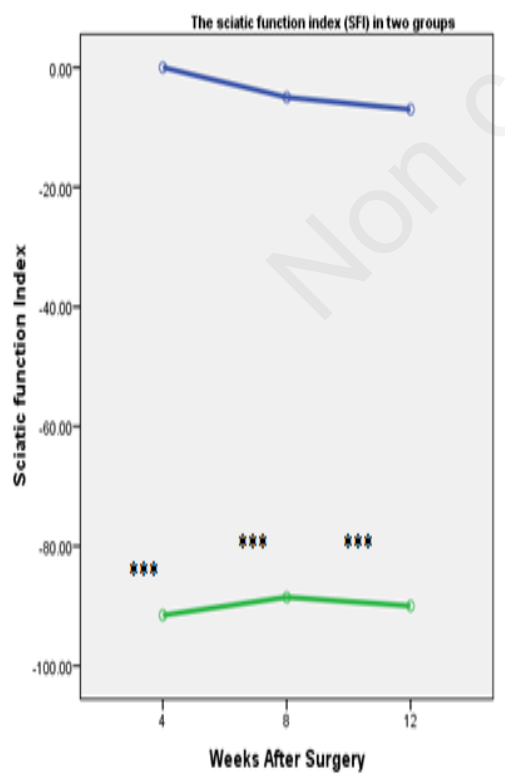

Fig 4. Sciatic function index. The significant difference between axotomy group and control group is indicated. $* * * p<0.001$

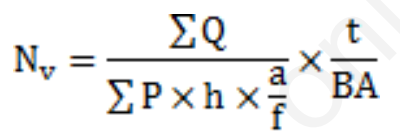

where $\Sigma \mathrm{Q}$ is the number of the nuclei, $\mathrm{h}$ is the height of the disector, $\mathrm{a} / \mathrm{f}$ is the frame area, $\Sigma \mathrm{P}$ is the total number of the unbiased counting frame in all fields, $t$ is the real section thickness measured in every field using the microcator, and BA is the block advance of the microtome which was set at $10 \mu \mathrm{m}$ and $5 \mu \mathrm{m}$ (for astrocytes cells). The total number of the neurons and astrocytes cells was estimated by multiplying the numerical density $(\mathrm{Nv})$ by the total $\mathrm{V}$.

$$
\mathrm{N}_{\text {total }}=\mathrm{N}_{\mathrm{v}} \times \mathrm{V}
$$

\section{Data Analysis}

Comparison between groups was made by one-way analysis of variance (ANOVA) followed by Tukey's multiple comparison test to analyze the difference. Walk data were analyzed using the two-way ANOVA method (repeated measures mixed model ANOVA). After twoway ANOVA, differences between curves/groups were

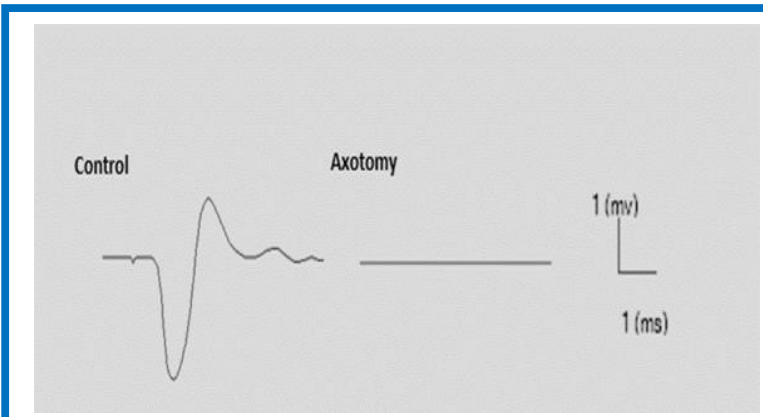

Fig 5. Muscle activity in rats. The sciatic nerve was stimulated, and the muscle action potential and latency were recorded in the gastrocnemius muscle. 
Table 2. EMG. Amplitude and Latency after 12 weeks post operation

\begin{tabular}{llll}
\hline Group & Control & Axotomy & p value \\
\hline Amplitude (mv) & $34.03 \pm 1.162$ & 0.00 & $<0.001$ \\
Latency (ms) & $1.00 \pm 0.089$ & 0.00 & $<0.001$ \\
\hline
\end{tabular}

All data were expressed as the mean \pm standard error of mean

determined by the Mann-Whitney test. All statistical work was performed in IBM SPSS version 21. All data are represented as the mean \pm SEM. The significance of the comparisons was set at $\mathrm{p}<0.05$.

\section{Results}

\section{Fuctional analyses}

Walking track analysis was performed to assess the eventual recovery of locomotive function in the experimental rats. At weeks 4,8 and 12, rats in the axotomy groups showed a time-dependent decrease in SFI values due to sciatic nerve degeneration and gastrocnemius muscle atrophy that were significantly different compared to control group $(\mathrm{P}<0.001)$ (Figure 4). At week12 post operation, results of the muscle EMG activity showed that compound action potential amplitude in gastrocnemius muscle was reduced in axotomy group compared with the control group. Difference was statistically significant $(\mathrm{P}<0.001)$. Significant differences in the latency after sciatic nerve injury were also seen between the 2 groups $(p<0 / 001)$, (Figure 5 and Table 2).

\section{Volume of spinal anterior horn}

After the sciatic nerve injury, there was a significant reduction in the total volume of spinal anterior horn in the axotomy group in comparison with the control group $(* \mathrm{P}<0.05)$ (Figure 6, A)

\section{Numbers of motor neurons and astrocyte cells}

Our stereological analysis showed that the total number of motor neurons was reduced in the axotomy group in comparison to the control group ( $\mathrm{P}<0.05$ ), (Figure 6, B). For detection of astrocyte migration (gliosis), immunohistochemistry against GFAP in the anterior horn of spinal cord was done. Migration of astrocytes in the axotomy group was statistically higher than that in the control. $(p<0.05)$ (Figure 7).

\section{Discussion}

When a peripheral nerve is cut, there is an immediate paralysis of the muscles and loss of sensation in the area supplied by the nerve. Our findings indicate, indeed, a time-dependent decreases of SFI values related to the sciatic nerve axotomy, accompanied by atrophy of the gastrocnemius muscle. Furthermore, EMG compound action potential amplitude was significantly reduced in the gastrocnemius muscle. These functional results are accompanied by the finding of the death of motor neurons in the anterior horn of spinal cord, where using immunodetection method, we demonstrated also that axotomy significantly increased astrogliosis.

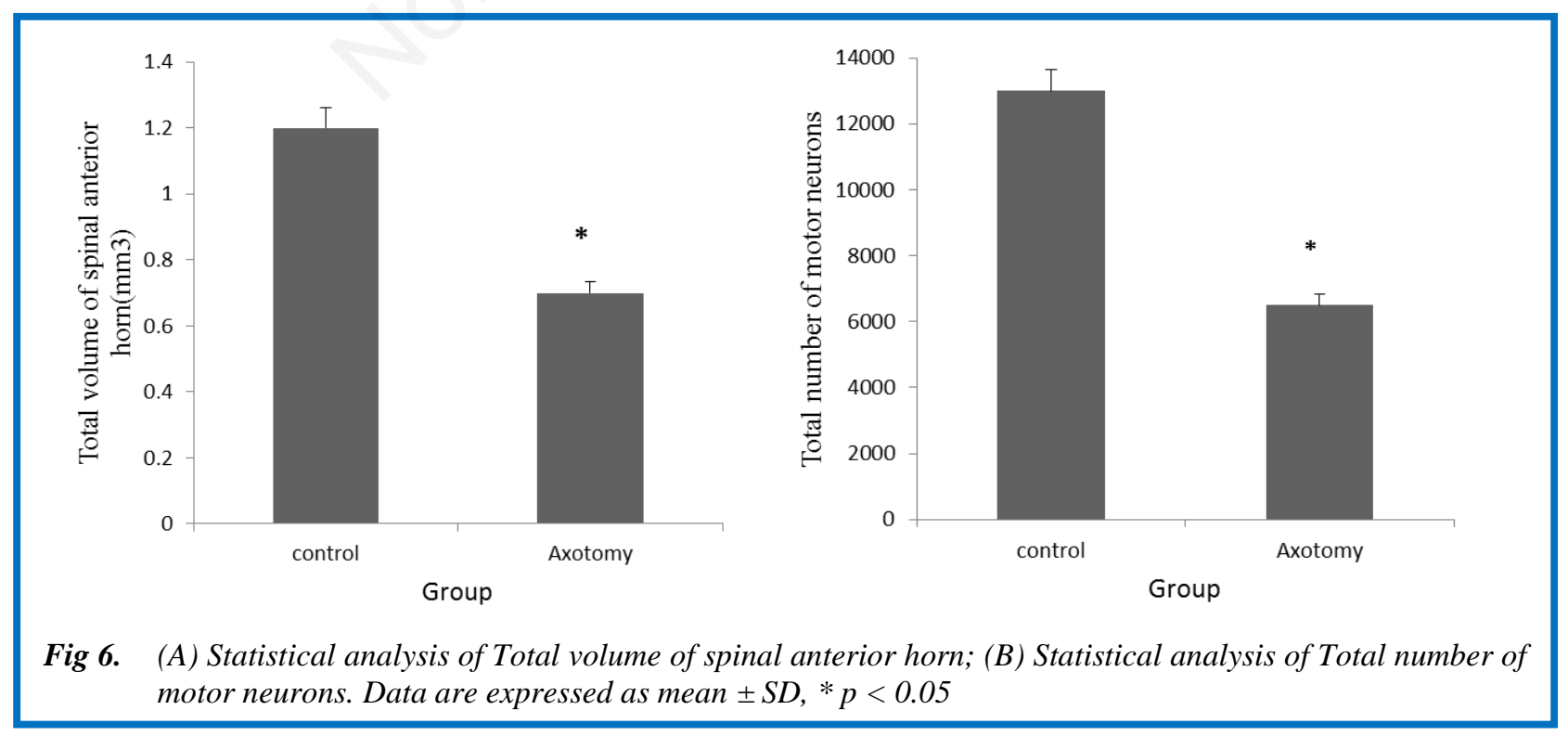




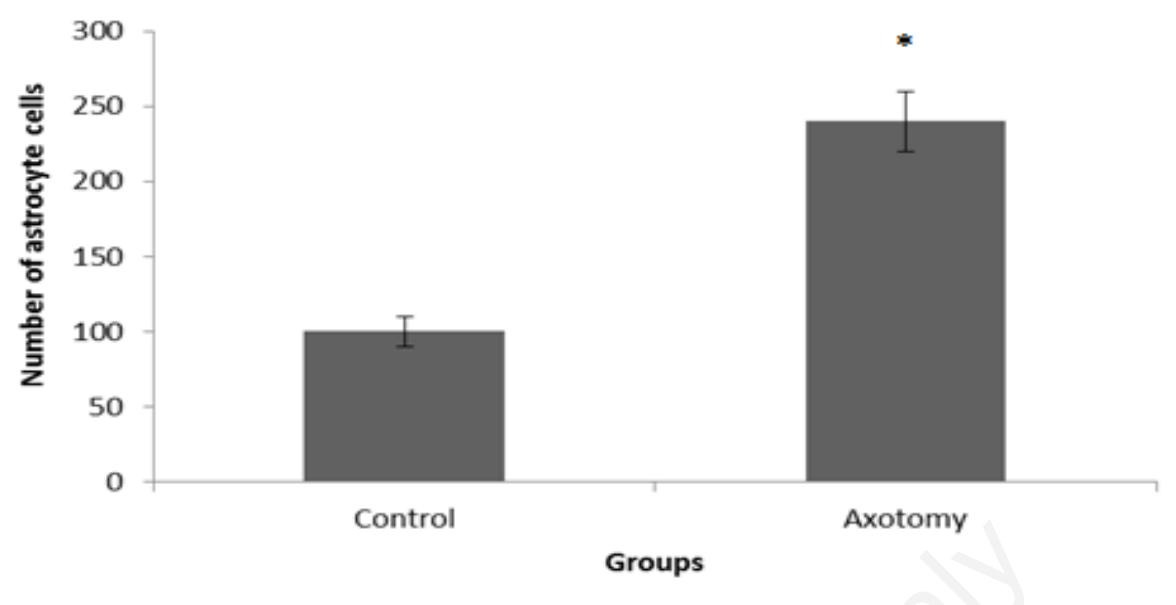

Fig 7. Statistical analysis of GFAP positive cells (astrocyte cells) in anterior horn of spinal cord. The significant difference between control with the axotomy groups is indicated. Data are expressed as mean $\pm S D$, *p< 0.05 .

In this study axotomy of the sciatic nerve induced both the expected impairment of motor function and of histological structure of spinal anterior horn. Specifically, the damage of the peripheral nerve is associated with neurodegenerative events in the spinal anterior horn. Axotomy has a very long experimental history as a paradigm to study responses of motor neurons to injury. ${ }^{17}$ Our results add that the disappearance of Nissl-stained profiles from the ventral horn reflects death of these cells and is not alterations of the staining properties of the injured motor neurons. Indeed, a significant reduction in total volume of the spinal anterior horn occurred in the axotomy group. Furthermore, by stereological analysis a statistically significant decrease of the motor neurons is present in the axotomy group.

Nervous system inflammatory responses occurring after nerve injury are initiated by peripherally derived immune cells, and activated glial cells that proliferate or migrate into the lesion site following injury. ${ }^{18,19}$

Many mechanisms may be involved in loss of anterior horn motor neurons. Soon after an axonal lesion, the process of Wallerian degeneration occurs in distal nerve fibers. ${ }^{20,21}$ In the distal stump, the cytoskeletal structures (neurofilaments and microtubules) disintegrate, with accumulation of granular debris in the axoplasm. Cell membrane disappear, the axon undergoes fragmentation and the myelin rapidly disintegrates. In rodents, these events usually occur between 2 to 3 days following axotomy ${ }^{22}$. There are also changes at the nerve cell body level occurring after distal nerve trauma. ${ }^{23}$ The most consistent and conspicuous observation in cell body following nerve injury is probably chromatolysis, that involves disintegration of the Nissl substance (large condensation of the endoplasmic reticulum). In addition, nuclear eccentricity, nucleolar enlargement and cell swelling can be noted. Other changes include increases in cytoplasmic acid phosphatase and smooth endoplasmic reticulum with hypertrophy of Golgi apparatus. The main metabolic event seems to be an increase in nuclear RNA synthesis, associated with an increase in cytoplasmic protein synthesis and content. ${ }^{24,25}$ Martin et al., suggested that chromatolysis is a form of apoptosis. Their study demonstrated that after 21 days of unilateral sciatic nerve avulsion in adult rats, the number of large motoneurons in the lumbar spinal cord was reduced by one third. ${ }^{26}$ Other studies have also shown that ventral root avulsions cause motor neurons death associated with neuronophagia. As a model of retrograde degeneration, the L4-L5 ventral rhizotomy is similar to axotomy paradigms in the CNS, in which loss of differentiated transmitter phenotype (usually an early change) is accompanied by alterations in the neuronal cytoskeleton and, eventually, cell death. ${ }^{27,28} \mathrm{Li}$ et al. reported that in adult rats, nerve avulsion induced a $70 \%$ loss by 3 weeks, whereas, by 6 weeks, all motor neurons were lost. ${ }^{29}$ Additional studies showed that the mode of motor neuron degeneration is complex and is related to developmental age and type of lesion. ${ }^{30}$ During the developmental cell death period, peripheral neurons are dependent on trophic factors produced by their targets cells, and those developing neurons that fail to compete successfully for sufficient target support die by apoptosis. ${ }^{31,32}$ 
In summary, based on present findings on adult rat, axotomy of the sciatic nerve is followed by: i) a timedependent decrease in SFI; ii) a marked decrease of EMG compound action potential amplitude in atrophying gastrocnemius muscle; iii) a remarkable reduction in the total volume of the spinal anterior horn; with iv) a reduction of the total number of motor neurons; and v) an increased presence of astrocytes in the anterior horn.

All together these quantitative analyses will be usefull to collect strong evidence of extent of functional changes and to test hypotheses and protocols for damage/repair mechanisms of motor neuron and their target organs in experimental and clinical research aimed to understand pathological processes and the eventual beneficial effects of interventions in peripheral nerve disorders and beyond. ${ }^{33-36}$

\section{List of acronyms}

EMG - Electromyography

PNI - peripheral nerve injury

SFI - Sciatic function index

\section{Author's contributions}

AR, MAA, AA, AP, FH, ES, ARR and YS equally participated in experimental design, data collection, writing and revision of the manuscript.

\section{Acknowledgments}

This article has been extracted from a PhD thesis and research project written by Mr. Amir Raoofi in the School of Medicine at Shahid Beheshti University of Medical Sciences (grant number: 11352).

Funding None.

\section{Conflict of Interest}

The authors report no conflicts of interests.

\section{Ethical Publication Statement}

We confirm that we have read the Journal's position on issues involved in ethical publication and affirm that this report is consistent with those guidelines.

\section{Corresponding Author}

Yousef Sadeghi, Department of Biology and Anatomical Sciences, School of Medicine, Shahid Beheshti University of Medical Sciences, Tehran, Iran.

Tel: +98-2122439976

Email: dr.ysadeghi@yahoo.com

\section{E-mails of co-authors}

Amir Raoofi: amirrezaraoofi@yahoo.com Mohammad Amin Abdollahifar: m_amin58@yahoo.com Abbas Aliaghaei: aghaei60@gmail.com

Abbas Piryaei: piryae@sbmu.ac.ir

Fatemeh Hejazi: mahsa.hejazi@gmail.com

Ensieh Sajadi: e.sajadi111@gmail.com

Ali Rashidiani-Rashidabadi:

ali.rashidian88@gmail.com

\section{References}

1. Grinsell D, Keating CP. Peripheral nerve reconstruction after injury: a review of clinical and experimental therapies. BioMed Res Int 2014;2014:698256. doi: 10.1155/2014/698256

2. Siemionow M, Brzezicki G. Chapter 8 current techniques and concepts in peripheral nerve repair. Int Rev Neurobiol 2009;87:141-72. doi: 10.1016/S0074-7742(09)87008-6.

3. Park KK, Liu K, Hu Y, et al. Promoting axon regeneration in the adult CNS by modulation of the PTEN/mTOR pathway. Science. 2008;322(5903):963-6.

4. Frade JM, Ovejero-Benito MC. Neuronal cell cycle: the neuron itself and its circumstances. Cell Cycle 2015;14(5):712-20. doi: 10.1080/15384101.2015.1004937.

5. Himes BT, Tessler A. Death of some dorsal root ganglion neurons and plasticity of others following sciatic nerve section in adult and neonatal rats. J Comp Neurol 1989;284:215-30.

6. Vestergaard S, Tandrup T, Jakobsen J. Effect of permanent axotomy on number and volume of dorsal root ganglion cell bodies. J Comp Neurol 1997;388:307-12.

7. Terenghi G. Peripheral nerve regeneration and neurotrophic factors. J Anat 1999;194:1-14.

8. Pierucci A, de Oliveira AL. Increased sensory neuron apoptotic death 2 weeks after peripheral axotomy in C57BL/6J mice compared to A/J mice. Neurosci Lett 2006;396:127-31.

9. Terenghi G. Peripheral nerve regeneration and neurotrophic factors. J Anat 1999;194:1-14.

10. Hyun JK, Kim HW. Clinical and experimental advances in regeneration of spinal cord injury. $\mathrm{J}$ Tissue Eng 2010;2010:650857.

11. Zalewski AA, Gulati AK. Rejection of nerve allografts after cessation of immunosuppression with cyclosporine A. Transplantation 1981;31: 8890.

12. Wiberg R, Kingham PJ, Novikova LN. A morphological and molecular characterization of the spinal cord after ventral root avulsion or distal peripheral nerve axotomy injuries in adult rats. J Neurotrauma 2017;34:652-60.

13. Wood MD, Kemp SW, Weber C, et al. Outcome measures of peripheral nerve regeneration. Annals of Anatomy-Anatomischer Anzeiger 2011;193: 321-33.

14. Bain JR, Mackinnon SE, Hunter DA. Functional evaluation of complete sciatic, peroneal, and posterior tibial nerve lesions in the rat. Plast Reconstr Surg 1989;83:129-38.

15. Ahmadi H, Boroujeni ME, Sadeghi Y, et al Sertoli cells avert neuroinflammation-induced cell death and improve motor function and striatal atrophy in rat model of Huntington Disease. J Mol Neurosci 2018;65:17-27. 
16- Noorafshan A, Abdollahifar MA, Asadi-Golshan R, Curcumin and sertraline prevent the reduction of the number of neurons and glial cells and the volume of rats' medial prefrontal cortex induced by stress. Acta Neurobiol Exp (Wars) 2014;74:44-53.

17. Nissl F. Uber die veranderungen der ganglienzellen am facialiskern des kanincheng anch ausreissung der nerven. Allg Z Psychiatr 1892;48:197-8.

18. Tian DS, Xie MJ, Yu ZY, et al. Cycle inhibition attenuates microglia induced inflammatory response and alleviates neuronal cell death after spinal cord injury in rats. Brain Res 2007;1135:177-85.

19. Kao CH, Chio CC, Lin MT, Yeh CH. Body cooling ameliorating spinal cord injury may be neurogenesis-, anti-inflammation- and angiogenesis -associated in rats. J Trauma 2010;70:885-93.

20. Preyat N, Rossi M, Kers J, et al. Intracellular nicotinamide adenine dinucleotide promotes TNFinduced necroptosis in a sirtuin-dependent manner. Cell Death Differ 2015:23;29-40.

21. Naidu M, David P. Major cellular events in peripheral nerve regeneration: A brief overview. Inter Med J 2009;8:69-72.

22. Ma M, Ferguson TA, Schoch KM, et al. Calpains mediate axonal cytoskeleton disintegration during Wallerian degeneration. Neurobiol Dis 2013;56:34-46.

23. Richardson PM, Miao T, Wu D, et al. Responses of the nerve cell body to axotomy. Neurosurgery 2009;65(4 Suppl):A74-9.

24. Lieberman AR. The axon reaction: a review of the principal features of perikaryal responses to axon injury. Int Rev Neurobiol 1971;14:49-124.

25. Lieberman AR. Comments on the fine structural organization of the dorsal lateral geniculate nucleus of the mouse. Anat Embryol (Berl) 1974;145:2617.

26. Martin LJ, Kaiser A, Price AC. Motor neuron degeneration after sciatic nerve avulsion in adult rat evolves with oxidative stress and is apoptosis. J Neurobiol 1999; 40:185-201.

27. Gage FH, Wictorin K, Fischer W, et al. Retrograde cell changes in medial septum and diagonal band following fimbria-fornix transection: quantitative temporal analysis. Neuroscience 1986;19:241-55.

28. Koliatsos VE, Applegate MD, Kitt CA, et al. Aberrant phosphorylation of neurofilaments accompanies transmitter-related changes in rat septa1 neurons following transection of the fimbriafornix. Brain Res 1989;482:205-18.

29. Li L, Wu W, Lin LF, et al. Rescue of adult mouse motoneurons from injury-induced cell death by glial cell line-derived neurotrophic factor. Proc Natl Acad Sci USA 1995;92:9771-5.

30. Li L1, Houenou LJ, Wu W, et al. Characterization of spinal motoneuron degeneration following different types of peripheral nerve injury in neonatal and adult mice. J Comp Neurol. 1998;396:158-68.

31. Otten U, Goedert M, Schwab M, Thibault J. Immunization of adult rats against 2.5 S NGF: effects on the peripheral sympathetic nervous system. Brain Res 2012;176:79-90.

32. Nakamura SI, Myers RR. Injury to dorsal root ganglia alters innervation of spinal cord dorsal horn lamina involved in nociception. Spine (Phila $\mathrm{Pa}$ 1976). 2000;25:537-42.

33. Willand MP. Electrical Stimulation Enhances Reinnervation After Nerve Injury. Eur J Transl Myol 2015;25(4):243-8. doi: 10.4081/ejtm.2015.5243. eCollection 2015 Aug 24. Review.

34. Carraro U, Kern H, Gava P, et al. Biology of Muscle Atrophy and of its Recovery by FES in Aging and Mobility Impairments: Roots and By-Products. Eur J Transl Myol 2015;25(4):221-30. doi: 10.4081/ejtm.2015.5272. eCollection 2015 Aug 24. Review.

35. Edmunds KJ, Gargiulo P. Imaging Approaches in Functional Assessment of Implantable Myogenic Biomaterials and Engineered Muscle Tissue. Eur J Transl Myol 2015;25(2):4847. doi: 10.4081/ejtm.2015.4847. eCollection 2015 Mar 11. Review.

36. Gargiulo P, Helgason T, Ramon C, Jr HJ, Carraro U. CT and MRI Assessment and Characterization Using Segmentation and 3D Modeling Techniques: Applications to Muscle, Bone and Brain. Eur J Transl Myol 2014;24(1):3298. doi: 10.4081/ejtm.2014.3298. eCollection 2014 Mar 31.

Submission: November 19, 2018

Revision received: December 17, 2018

Acceptance: December 17, 2018 